\title{
UNA APROXIMACIÓN AL DESARROLLO RURAL DE COSTA RICA. LA EXTENSIÓN AGRÍCOLA: BREVE SÍNTESIS DE SU EVOLUCIÓN Y TENDENCIAS HACIA SU DEMOCRATIZACIÓN
}

\author{
Dr: Fernando Rivera $R^{*}$
}

En Costa Rica. la extensión agricola ha sido un imporrante instrumento de desarro. Ilo rural. El documento nos presem u una inliosa serie de caraclerislicas de la exlension agricola a pariir del sigio XIX. señulando hiros ligados a los modelos de desarrollo imperantes en diferentes momentos de la his. coria instinucional del pais.

Desde esta perspectiva de la exiension agricola-y sobre lodo de las caraclerislicas que esia revistió desde los aitos90- el auror propone seis lineas de acción con miras al desarrollo rural, que pasan por la democra. rización de la extensión y el replanteamiento de la investigación y la expension agricola dirigida a pequeños y medianos agriculıores.
In Cosla Rica, agrarian outreach programs have been em imporiant insmiment for rural development. This study presents a valuable set of characteristics of the programs carried out since the 19" century. and points ont achievements related to the development models existing at differens limes in the institurional histon of this commery: From this perspective of agrarian outreach. (nnd in particular: of she characteristics that is has had since the 1990s, the author has proposed six lines of actionfor rural development. They include the clemocratization of outreach programs and a new approach loward research and agrarian oulreach, oriented coward small and medium farmers.

- Doctor en Educación y Desarrollo Profesional por la Universidad East Anglea. Inglaterra. Posec una maesına en Educación Agrícola de la Uni versidad de Reading, en esc mismo país. Es ingeniero agróno mo por la Universidad de Cosia Rica y bachiller en Enseñanza de la Tecnología por la Universidad Nacional. Actualmente. es director de la Escuela de Ciencias Agrarias de la UNA. ferivera @e una.ac.c 


\section{Presentación}

El abordar el desarrollo rural en Costa Rica, en un seminario sobre Educación y Desarrollo Rural como el que se ha organizado, permite concentrar la atención en la Extensión Agrícola, por cuanto ha sido un componente importante de las políticas, programas y proyectos que diversas instituciones han impulsado en los espacios rurales, principalmente el Ministerio de Agricultura y Ganadería (MAG). Por otra parte, la definición de lo rural en la actualidad incluye actividades agropecuarias y no agropecuarias, cuyo análisis conduciría a tener que hacer generalizaciones sin poder profundizar adecuadamente en experiencias relacionadas con la educación rural, como se propone este seminario.

Por estas razones esta exposición se centrará en algunos aspectos de la evolución de la extensión agrícola y sus tendencias recientes, para contar con una referencia importante para entender el desarrollo rural en Costa Rica. Además, la extensión agrícola está vinculada directamente con la educación rural, por cuanto se propone generar cambios en la población rural, tanto en sus actividades productivas en la finca, como en aspectos comunitarios donde los adultos son los protagonistas, y requiere de la aplicación de principios y actividades de enseñanza y aprendizaje.

Este documento sintetiza algunos aspectos importantes de la extensión agrícola en Costa Rica, cuya consideración puede contribuir a mejorar ese servicio, aunque con este breve análisis no se pretende agotar cl tema planteado. Con el fin de facilitar la exposición, el documento resalta puntualmente su contenido.

\section{Introducción}

El desarrollo rural se puede definir como las condiciones y la calidad de vida de la población en los espacios rurales en un determinado momento de su historia. Si bien se plantea con este concepto una dicotomía entre lo rural y lo urbano, hay factores y aspectos comunes que influyen y condicionan el desarrollo rural y urbano. Así, por ejemplo, las políticas educativas parten de los mismos principios y objetivos aun cuando necesariamente se ajusten a aspectos particulares de la población involucrada, sea ésta rural o urbana.

Es por ello que en la actualidad, el tratamiento de lo rural no puede omitir el análisis de los estilos o modelos de desarrollo que predominan en la sociedad, sus paradigmas y bases teóricas y metodológicas, y sus efectos. No obstante, hay una mutua condicionalidad entre lo urbano y lo rural; al velar los grupos y sectores rurales por sus intereses, influyen en las decisiones que el gobierno toma al impulsar, por ejemplo, determinadas políticas alimentarias que afectan 
también a la población urbana. En la actualidad, los límites o diferencias entre lo rural y lo urbano son menos marcados. Se implantan industrias no agrícolas en zonas rurales, y éstas contienen servicios y actividades que anteriormente eran típicas de las zonas urbanas.

Las políticas macroeconómicas que fundamentan cl proceso de desarrollo vigente en Costa Rica asumen, entre otras consideraciones, que el mercado es el llamado a regular la sociedad sin intervención del Estado, por medio de las entidades de gobierno y de otras esferas de poder como la sociedad civil. Desde mediados de los años 80 estas políticas han tenido un papel detcrminante, al modificar las políticas agropecuarias y han incidido en la situación de la población rural, lo cual demuestra la depeiidencia de lo que ocurre en estos espacios con respecto a las políticas de desarrollo nacional.

El concepto 'desarrollo' supone mejoramiento, progreso, lo que implica cambio social. Sin embargo, no siempre se logra mejorar las condiciones de vida por cuanto los cambios que el desarrollo genera no necesariamentc son beneficiosos. El desarrollo cs un proceso sujeto al papel de las fuerzas sociales que procuran mejorar sus condiciones de vida: sus intereses, contradicciones, acuerdos o desacucrdos son parte importante de la definición de las políticas de desarrollo nacionales e internacionales y de su materialización, proceso en el cual se combinan aspectos sociales, políticos, económicos, culturales, ambientales, y del tipo de recursos naturales disponibles, su calidad y cantidad, que le dan sustento a la población. Estos aspectos hacen que el desarrollo rural sea complejo y diferenciado, y su análisis se ha enriquecido al incorporar el tratamiento de cuestiones regionales y locales, como ámbitos sociales y espaciales más integradores para su estudio y definición de políticas (MDR, 1996).

La extensión agricola ha sido un instrumento y/o estrategia de desarrollo rural (Fernández y Rivera. 1991). Ha tenido la responsabilidad de introducir y desarrollar nuevas técnicas de producción, de cultivos y especies animales, y ha servido para generar otros requerimientos sociales, como la investigación agropecuaria, la comercialización y obras de infraestructura como caminos y clectrificación. También ha acompañado cambios en las actividades de los espacios rurales al responder con la incorporación de actividades de conservación y manejo de recursos naturales como los bosques y el agua, la agroindustria, y hay iniciativas de agroturismo que demandarán el apoyo de la extensión al ampliarse las formas en que la población rural se involucra en el desarrollo local y regional. 


\section{Breve caracterización de la extensión agricola en Costa Rica}

3.1. La extensión agrícola es un scrvicio que el MAG ha ofrecido desde 1956. el cual se inició en 1949 como resultado de acucrdos previos tomados por los gobiernos de Costa Rica y los Estados Unidos a partir de 1942. Surgió para acopiar alimentos para las fucrzas militares de los Estados Unidos en el Canal de Panamá, y se orientó luego al mejoramiento de la producción por medio de la capacitación de agricultores en el manejo de agroquímicos y la introducción de diversas técnicas de producción (Arroyo, 1953). No obstante, previamente en el país, desde mediados del siglo XIX, diversas instituciones educativas llevaban a cabo actividades que pucden ser catalogadas como de extensión, los niveles de educación secundaria, técnica (parauniversitaria) y universitaria, así como por iniciativa de otras gubernamentales (Sáenz, 1978: Sáenz, 1985).

3.2. Al asumir el MAGeste servicio, se abricron, paulatinamente agencias de extensión, las cuales contribuyeron a mejorar las condiciones de vida en los espacios rurales al incidir en la tecnología agropecuaria. Las visitas a finca, la demostración de resultados y de método, las reuniones y cursos, eran técnicas importantes con que los extensionistas llevaban a cabo su trabajo. Desde sus inicios, la extensión en Costa Rica aplicó principios de educación derivados de la experiencia y enfoques de universidades cstadounidenses que apoyaron su desarrollo en América Latina. Esto significa que, este servicio, se propuso impulsar cambios en la producción por medio de la cducación, del aprendizaje y la enseñanza, lo que caracteriza sus fundamentos filosóficos (Rice, 1974; Campos, 1988).

3.3. Se decretó la regionalización del servicio de extensión por medio de direcciones en cada región, que al inicio de los 70 fueron fortalecidas con la construcción de instalaciones y más personal. Esto hizo posible una mayor estabilidad de los extensionistas al disponer de mejores condiciones de trabajo, y al constituirse equipos de profesionales que en cada región se responsabilizaron por la investigación y el apoyo técnico al personal de las agencias de extensión. Con la fundación de la Universidad Nacional (UNA), se impulsa a partir de 1974, la extensión que se incorpora como parte de su quehacer académico y se impulsan proyectos que introducen enfoques participativos y varios curricula incluyeron actividades en las comunidades rurales, como las convivencias con familias campesinas en el caso de los agrónomos (Fernández y Rivera, 1991); estas experiencias coincidieron con planteamientos como los de Freire (1972).

3.4. En esta década se introdujeron enfoques que fueron priorizando aspectos tecnológicos y productivos, por ejemplo, con el impulso del Programa de Incremento de la Productividad Agropecuaria (PIPA) (Cordero, 1986); estos 
En la actualidad, los límites o diferencias entre lo rural y 10 urbano son menos marcados. Se implantan industrias no agricolas en zonas rurales, y éstas contienen servicios y actividades que anteriormente eran típicas de las zonas urbanas. enfoques estaban relacionados con directrices emanadas de acuerdos de los gobiernos con organismos internacionales de financiamiento, como el BID, el Banco Mundial. Por ejemplo, entre 1978 y 1982 se introdujo el método de capaciración y visiras (C. y V.) (Bolaños, 1990); este enfoque modifico profundamente la extensión, tanto en su organiza-

ción como en su filosofía, y fue desechado por haber reducido la cobertura social del servicio.

3.5. Simultáneamente, se fue instaurando una cultura política e institucional donde los nombramientos para diferentes pucstos en el servicio, eran matizados por los intereses políticos e ideológicos de los partidos políticos en el gobierno. Esto, a su vez, se fue polarizando con los cambios de administración de cada gobierno, en el sentido de que las políticas agropecuarias del MAG se han venido modificando, al menos desde los años 70, al tenor de las directrices del gobierno de turno, sus ministros de agricultura y los asesores.

3.6. A partir del inicio de los años 80 , se impulsaron orientaciones sobre las políticas de investigación y extensión del MAG, con el apoyo del ISNAR, tendientes a articular ambas áreas de trabajo, por cuanto estaban separadas y se presentaban desfases en su implantación al tratar de que los agricultores pudiesen incorporar los resultados de la investigación (Osten et al., 1981; ISNAR/ IICA, 1981; ISNAR/IICA, 1988). No obstante, la separación entre la investigación y la extensión todavía se mantiene.

3.7. Los lemas de las campañas electorales hacían cada vez más referencia a la población rural --'volvamos a la tierra' de 1982 a 1986, 'agricultura de cambio' de 1986 a 1990 y otras---, que recurrian a significados que creaban esperanza e ilusiones, mientras que en la práctica, en la realidad de los pequeños y medianos agricultores, la tendencia era a suprimir los servicios. Es en estos períodos que se implantan programas de ajuste estructural (PAE) a partir de 1985, que en general son valorados positivamente en la documentación oficial, y tienden, entre otros aspectos, a la privatización de los servicios gubernamentales (Villasuso, 1992; MIDEPLAN, 1993).

3.8. No obstante lo anterior, su situación de ruina la vienen planteando los pequeños y medianos agricultores en relación con los efectos de las políticas macroeconómicas y agrícolas, que comenzaron a implantarse desde mediados de $\operatorname{los} 80$, algunos de los cuales han sido sistematizados (Aguilar et al., 1988; 
CENAP, 1989; Vermeer, 1990; Cartín, 1990; Rivera, 1991). Además, los han cuestionado por saber que en los países desarrollados se mantienen diferentes mecanismos de subsidio e incentivo para la agricultura, que los organismos financieros internacionales y nuestros gobiernos han estado eliminando, lo que los pone en condiciones de mayor vulnerabilidad. Estos efectos los ha conducido a protestar, a hacer blogueos y presentar recursos legales contra las políticas de apertura (LN 16-6-00:6 A; LN 28-7-00:4 A; LN 8-8-00:4 A; LR 26-10-00:6 A). Por otro lado, se dispone de datos que dan cuenta de la disminución de pequeños agricultores y los niveles de pobreza rural (Proyecto Estado de la Nación, 1997).

3.9. Las políticas agrícolas y de desarrollo que se han aplicado se orientan a excluir a estos agricultores (Trejos, 1998). Los cambios en el quehacer de las instituciones del sector agropecuario han contribuido a desarrollar un panorama diferente en los espacios rurales, que tiene incidencias profündas y requiere tomarlos en cuenta para el desarrollo de una nueva institucionalidad (Femández y Granados, 1999). Esta situación tiene efectos importantes en la extensión, por cuanto es a esa población que dirige sus servicios, por lo que es necesario que la extensión y los pequeños y medianos productores asuman un papel más activo en la definición de las políticas agrícolas que les incluya como sujetos sociales que puedan mejorar sus condiciones de vida. Al respecto Engel (1998) indica que la extensión está en crisis como resultado, entre otros aspectos, del proceso de globalización y privatización.

\section{Aproximación a la situación actual de la extensión}

\subsection{La voz de algunos actores importantes}

\section{Convenio CNP - Extensión MAG ... Un Fracaso}

El sector agrapecuario del país, desde hace muis de una década, viene experimentando una importante crisis de orientación. Desde la aplicación de los programas de ajuste estructural, los pequeños y medianos productores agropecuarios y foresta. les, pasan por una situación incierta y crítica, pues no logran encontrar caminos claros y seguros para insertar sus productos en los mercados de una manera exitosa.

Esta situación se ha visto agravada por la forma en que se vienen conduciendo las instituciones publicas del sector; sometidas a grandes restricciones presupuestarias y 'reformas' improvisadas, que en nada han contribuido al mejoramiento de los servicios al pequeño productor:

... Kistóricamente ha faltado liderazgo ministerial y cada jerarca e institución tiende a comportarse como un ente aislado, contribuyendo con ello a generar mayor confusión y duplicidad en el uso de los escasos recursos. 
Ante la incapacidad de estructurar instituciones novedosas y en concordancia con los importantes procesos de desarrollo tecnológico y gerencia que se viven en la actualidad, el Ministerio de Agricultura y Ganaderia estableció un convenio con el Consejo Nacional de Producción para integrar los servicios que se ofrecen al agricultor. Un año después de haberse llevado a la práctica dicho convenio, los extensionistas han observado los siguientes resultados:

1. No se ha dado la integración de servicios planteada en el convenio, por el contrario, se ha desvirtuado el concepto de extension, deteriorado el servicio en cobertura, calidad y capacidad de respuesta.

2. Lo anterior ha provocado una desmotivación, desconfianza e incertidumbre en el beneficiario del servicio y en sus funcionarios.

3. Las incompatibilidades legales e institucionales, implicitas en el convenio. han llevado a un mayor entrabamiento en la prestacion de los servicios, y tienen además consecuencias sobre su sostenibilidad instimcional.

4. El progresivo desfinanciamiento del servicio de extension se ha visto agravado por la ausencia de estabilidad financiera del CNP.

5. En vez de agilizar la acción institucional, se incrementó el número y tipo de requisitos burocráticos para realizar todo tipo de gestión.

Tomando en cuenta los resultados anteriores, provenientes de evaluaciones realizadas por la Secretaría de Planificación Sectorial Agropecuaria. Dirección de Planificación del Consejo Nacional de Producción. Dirección de Planificación Estratégica del Ministerio de Agricultura y Ganaderia, Direc. ción de Extensión y las organizaciones sindicales del MAG. llamamos la atención del máximo jerarca y rector del sector agropecuario, Dr: Alberto Dent Zeledón. para que tome la decisión de rescindir el convenio.

Por otra parte, manifestamos nuestra disposición en participar de acciones co. herentes y planes sectoriales bien estructurados que conduzcan al mejoramiento de los senvicios brindados a los productores agropecuarios en aspectos tecnológicos. comerciales, agroindustriales y organizativos. La integracion debe sustentarse en proyectos productivos de las onganizaciones de productores con apoyo de equipos técnicos interinstimcionales y multidisciplinarios de común acuerdo con los agricultores.

Asociación de Extensionistas Agropecuarios y Forestales (ANEAF)

Unión de Empleados del MAG y del MINAE (UDEMAG-MINAE)

Unión de Técnicos del MAG y del MINAE (UTAMAG-MINAE)

Unión de Secretarias y Oficinistas del MAG (USOMAG)

Tornado de LN 6-10-2.000:18 A. 


\subsection{Algunos otros efectos de los cambios de las políticas agricolas que conforman el contexto de la extensión}

4.2.1. Al tomar más importancia las políticas macroeconómicas del desarrollo nacional e internacional, el desarrollo rural ha trascendido lo meramente agropecuario por cuanto la población rural se dedica también a estas actividades. Por ello se requiere incluir otras actividades como la forestal, la conservación y de recursos naturales -incluida la vida silvestre-, la agroindustria, y otras que constituyen servicios que en los espacios rurales se necesitan. Esto significa la importancia de que la extensión asuma también actividades como éstas, lo que plantea una mayor complejidad para los extensionistas y las instituciones que trabajan en la capacitación con comunidades rurales.

4.2.2. Los cambios que se han presentado en los espacios rurales de Costa Rica, desde la mitad de los años 80 , han obedecido fundamentalmente a las políticas de reestructuración de la economia y de reforma institucional que han aplicado los gobiernos. Se han aducido acuerdos internacionales para dirigir la economía hacia una globalización que supuestamente es necesaria, con base en su desregulación, apertura e internacionalización, donde la capacidad de los estados como naciones pierden funciones y atribuciones en aras de que la economía de mercado sea el único ente regulador de las relaciones entre los diversos sujetos y actores del desarrollo. Estas orientaciones del desarrollo han impactado negativamente a las instituciones encargadas de formular y ejecutar políticas agrícolas y de desarrollo rural, como el CNP, el MAG y el IDA, al restringir sus funciones y a los agricultores.

4.2.3. Las modificaciones de las políticas agrícolas han tenido un impacto considerable, como por ejemplo: a- la reducción de servicios dirigidos a productores de granos básicos -crédito, asistencia técnica, apoyo en la comercialización--; b- el traslado de algunas funciones que eran de entidades públicas, a manos de entidades privadas, como la gestión de varios componentes del sector cafetalero que anteriormente se impulsaba en cooperación con el MAG, o el abastecimiento de los granos básicos; c- la eliminación de un número considerable de plazas en el sector público agropecuario como se indica en el Cuadro 1: 


\section{Cuadro 1 \\ Reducción de la planilla en varias instituciones \\ agropecuarias (1991-1997)}

\section{INSTITUCIÓN}

Ministerio de Agricultura

y Ganadería (MAG)

Consejo Nacional

de Producción (CNP)

Instituto de Desarrollo

Agrario (IDA)
PLAZAS VARIOS AÑOS

1994: 1.854

1997: 1.167

199i: 2.756

1997: 656

1995: 809

1997: 476
REDUCCIÓN

Absoluto: 687

$37 \%$

Absoluto: 2.100

$76 \%$

Absoluto: 333

$41 \%$

Fucnte: Fernéndez, Af. y Ara) a. G, 1999, «Anślisis theliuninar sobre la Mosemización del Sector Esıałal Agropecuasio en Cusla Rica y su Repercusión en los Servicios al Productons. Inssituto de Investigaciones Sociales. UCR. San José. Costa Rira.

4.2.4. En la extensión se aplicó un cambio importante en sus políticas a partir de setiembre de 1999 al trasladarla al CNP (LN 6-10-00:18 A.) Esto ha significado una mayor separación de la investigación, lo cual contradice diversos plantcamicntos y políticas del MAG tendientes a articularlas.

4.2.5. La reestructuración y reforma institucional han inestabilizado la cxtcnsión, han repcrcutido en forma negativa psicologica y socialmente en cl personal que ha estado expuesto a esos cambios al generar incertidumbre, desmotivación y falta de orientación. Por otra parte, el proceso de cambio y de aprendizaje en los agricultores requicre estudiarse desde diversas árcas del conocimiento, debido a su complejidad, para supcrar una concepción mecanicista que a veces impera en la extensión y que apunta únicamente al cambio tecnologico. Esta situación también ha repercutido en los agricultores: uno de ellos explica con su testimonio la cuestión del cambio asi:

"No nos resistimos al cambio. lo que nos da miedo es hacer un cam-

bio mal hecho» (Alexis Bermúdez, 1999).

4.2.6. La reconversión productiva se ha convertido prácticamente en el eje de las políticas agrícolas -entre ellas las de extensión-, que sc presenta como la panacea. Sin embargo, en la práctica se ha centrado en el financiamiento, cuestión que si bien es importante, no es suficiente para mejorar la calidad de vida de los pequeños y medianos productorcs. Más prcocupante es aún que los fondos de ese programa se han concentrado en 8 proyectos (LR 17-10-00:5 A): esto plantea interrogantes sobre sus posibilidades reales de que cste programa 
ofrecezca una respuesta adecuada como parte de una estrategia de desarrollo rural integral, tanto regional como nacional.

\subsection{Iniciativas importantes de la extensión a partir de los 90}

4.3.1. Desde inicios de los 90 se impulsaron cambios relevantes en sus políticas, como el énfasis en la organización de los agricultores, el enfoque INVEX (Investigación-Extensión) y la extensión participativa, en cuya definición tuvieron un papel fundamental la Dirección de Extensión del MAG. Estos planteamientos y experiencias constituyen un aporte importante de funcionarios nacionales, que recogen sus experiencias y el ejercicio profesional con los agricultores, y que significa un rompimiento con la tendencia institucional a depender de manera importante de los planteamientos de organismos externos, tanto con respecto a aspectos metodológicos, como conceptuales.

4.3.2. Como resultado de múltiples factores y coincidencias, se desarrollaron varios proyectos con el enfoque de agricultores experimentadores en la Región Brunca a partir de 1993, como parte del Programa de Reforzamiento de la Investigación Agronómica en Granos Básicos (PRIAG) (Hernández. 1994), experiencia local e institucional que ofrece importantes aprendizajes para la investigación y la extensión (Bermúdez, Hernández y Rivera, 1999). Entre los aspectos convergentes de esta experiencia están:

a. la participación de varios extensionistas, con una formación y una práctica profesional vinculadas a pequeños y medianos productores -varios de ellos egresados de lá Escuela de Ciencias Agrarias de la UNA-, quienes junto con algunos investigadores, tuvieron la ética, la sensibilidad social y la visión de un desarrollo rural altemativo, para incorporar activamente a los agricultores en el desarrollo de experimentos y de la gestión de los proyectos:

b. la existencia previa de organizaciones de base de productores, que habían sido incentivadas por las políticas de extensión del MAG, lo que potenció el surgimiento de capacidades locales;

c. la participación de agricultores cuya práctica y aprendizaje se han basado en sus propios ensayos y experimentos, en relaciones de colaboración, apoyo mutuo e intercambio de conocimientos:

d. el papel de facilitador y promotor que desempeñó uno de los asesores externos del PRIAG, junto con algunos coordinadores nacionales. 
4.3.3. Cabe resaltar del enfoque agricultores experimensadores, los siguientes logros:

a. se articuló la investigación y la extensión como parte de un solo proceso de desarrollo local, combinando aspectos tecnológicos, políticos, sociales y económicos, a partir de decisiones compartidas entre agricultores, extensionistas e investigadores;

b. los agricultores tomaron la iniciativa y los funcionarios asumieron un papel de facilitadores, creándose relaciones intersubjetivas de colaboración y apoyo mutuo, superándose o al menos disminuyéndose el papel unilateral y un tanto arbitrario que tradicionalmente ocurría al mantener los profesionales el control sobre las preguntas fundamentales, como ¿qué investigar y por qué?;

c. la organización de los agricultores ha sido determinante en la ampliación de la cobertura de la investigación y la extensión, en la introducción de cambios tecnológicos y el mejoramiento de la capacidad y poder de negociación de los agricultores frente a los intermediarios y comerciantes;

d. se produjeron cambios cualitativos de vital importancia, como: d1. mejorar la interacción entre los diferentes actores, d2. valorar tanto el conocimiento popular de los agricultores como el de los profesionales como partes necesarias de las transformaciones que pueden implantarse en los espacios rurales, d3. amalgamar la investigación y la extensión a partir de la situación real de los agricultores de manera que ellos y su situación son el punto de partida y de llegada, d4. dar sentido al quehacer de la investigación y la extensión dirigidas a los pequeños y medianos productores, y de esta manera, construir opciones que contribuyan a mejorar sus condiciones de vida, d5. mostrar que lo tecnológico y el conocimiento científico son importantes, pero no necesariamente suficientes, por lo que la extensión requiere trabajar también con aspectos sociales, económicos y culturales de la población, lo que sustenta el planteamiento de que la extensión no es solo una cuestión de agrónomos, se requieren equipos multi e interdisciplinarios.

Esta experiencia y otras que es necesario rescatar y sistematizar sirven de referencia para construir opciones que fortalezcan la extensión, para que continúe apoyando a los pequeños y medianos agricultores, especialmente a quienes enfrentan condiciones de pobreza y a los que luchan porque su cultura sobreviva. 


\section{Propuestas para mejorar la extensión y su aporte al desarrollo rural}

5.1. Recuperar y sistematizar experiencias de extensión que permitan enriquecer su desarrollo retomando principios filosóficos y educativos, de manera que responda a la transformación necesaria que están demandando los pequeños y medianos productores, y que es posible construir con el apoyo de la extensión y de investigaciones que ayuden a retomar y reconstruir teorías del cambio y el aprendizaje que incluyan los aportes de los profesionales y agricultores y les apoye en la gestión del desarrollo rural.

5.2. Despolitizar el quehacer de las políticas de extensión y las de investigación, en el sentido de reducir la influencia de los partidos políticos y de los políticos, en su diseño y ejecución. llevando a la práctica la regionalización y descentralización de las políticas y sus instituciones.

5.3. Democratizar el desarrollo, las instituciones, las políticas y sus programas y proyectos, y propiciar la democratización de la extensión, en aspectos como: a- la estructura y organización institucional, de manera que las decisiones respondan a las necesidades de los grupos y sectores a quienes dirigen sus servicios, así como al aporte de técnicos y profesionales que trabajan con ellos: $b$ - la participación de los extensionistas y los agricultores en la definición de políticas, los programas y proyectos tanto de extensión como de investigación.

5.4. Replantear la investigación y la extensión dirigidas a los pequeños y medianos agricultores, a partir de su propia realidad y del desarrollo de proyectos y programas en que ambas se combinan como un solo proceso de desarrollo agrícola y rural, incluyendo la experimentación campesina. También es necesario fortalecer la investigación agropecuaria que instituciones y centros especializados deben llevar y articularla con la extensión. Esto significa un trabajo conjunto de investigadores y extensionistas con la participación de equipos inter y multidisciplinarios (con profesionales en agronomía, forestales, biología, economía, administración, sociología, pedagogía, antropología, entre otros, según se requiera apoyo técnico en la producción, la administración y la gestión del desarrollo local).

5.5. Construir una nueva ruralidad que tenga como apoyo importante la construcción de una nueva institucionalidad, con mayor autonomía e independencia del gobierno central en el diseño, ejecución y evaluación de sus actividades, con planes de mediano y largo plazo y estrategias participativas, y rendición de cuentas para con los sujetos del desarrollo rural. Cabe subrayar la importancia de que se considere crear una institución autónoma de desarrollo rural que articule las actividades dispersas y que se duplican entre instituciones, 
con base en el fortaleciendo de instancias de dirección regionales que incorporen a la población rural en la toma de decisiones.

\section{Referenicas}

Aguilar, A. et al. (1988) No hay paz sin alimentos. Los pequeños produçores por el derecho a producir. Litografía COMARFIL. CENAP/CEPAS/Justicia y PazJUNA. San José, Costa Rica.

Arroyo, C. (1953) Origen y desarrollo de la extensión agricola en Costa Rica. Tesis, Universidad de Costa Rica. San José, Costa Rica.

Bermúdez, A., Hernández, J.C. y Rivera, F. (1999) No nos resistimos al cambio, lo que nos da miedo es hacer un cambio mal hecho. PRIAG, San José, Costa Rica.

Bolaños, L. (1990) "La Experiencia Costarricense en Transferencia y Adopción de Tecnología por el Método de Capacitación y Visitas C. y V.". En Seminario Mobilizing Agricultural Techmology to Meet Central American Challenges. IICA, San José, Costa Rica.

Campos, G. (1988) Historia de la Extensión Agricola en Costa Rica. Origen y Desarrollo. Documento preparado con motivo del 40 aniversario de la Extensión Agrícola en Costa Rica. MAG, Costa Rica.

Cartín, S. (1990) Lucha social en el agro costarricense (La experiencia de UPIAV). Imprenta La Jornada. CEPAS, San José, Costa Rica.

Cordero, N. et al. (1986) Documento Central: Metodología de Seguimiento y Evaluación. SEPSA/MAG/IICA, San José, Costa Rica.

Engel, P. (1998) «Facilitando el Desarrollo Sostenible: ¿Hacia una Extensión Modema?» En ALARCÓN et al. (comp.) Situación Actual y Perspectivas del Complejo Transferencia de Tecnología, Asistencia Técnica y Extensión Agropecuaria. Memorias del Taller. IICA, San José, Costa Rica.

Femández, M. y Araya G. (1999). Análisis Preliminar sobre la Modemización del Sector Estatal Agropecuario en Costa Rica y su Repercusión en los Servicios al Productor. Instituto de Investigaciones Sociales, U.C.R., San José, Costa Rica. 
Fornández, L. y Granados, E. (1999) El impacto de las reformas estructurales y politicas macroeconómicas en el sector silvoagropecuario de Costa Rica. Documento para la División Agrícola de la CEPAL Chile.

Fernández, L. y Rivera, F. (1991) Administración en Extensión Rural. EUNED. San José, Costa Rica.

Freire, P. (1972) Extensión o comunicación: la concientización en el medio rural. Siglo XXI Editores. México.

Hernández, J.C. (comp. y edit.) (1994) Resuimenes de las ponencias del 1: Encuentro de Productores Experimentadores de la Región Pacífico Sur. MAG, Costa Rica.

Osten, A. et al. (1981) El Sistema de lnvestigación Agropecuaria y Tramsferencia de Tecnologia en Cosıa Rica. ISNAR, La Haya, Holanda.

Proyecto Estado de La Nación (1997) Estado de la Nación en Desarrollo Humcuno Sostenible. Imprenta EDITORAMA S.A. San José, Costa Rica.

ISNAR/IICA (1988) Forralecimiento del Sistema de Investigación Agropecuaria y Transferencia de Tecnologia. ISNAR, La Haya, Holanda.

ISNAR/IICA. (1988) Prioridades, Formación de Programas Nacionales y Programación de la Investigación y Transferencia de Tecnologia Agropecuaria. ISNAR, La Haya, Holanda.

MIDEPLAN (1993) Costa Rica: Balance del Ajuste Estructural. MIDEPLAN, San José, Costa Rica.

Maestria en Desarrollo Rural (MDR) (1996) Modificación Curricular. Universidad Nacional, Heredia, Costa Rica.

Rice, E.B. (1974) Extension in the Andes. Massachussets Institute of Technology Press, London.

Rivera, R. (1991) Lucha social en el agro costarricense: Las organizaciones campesinas de la Región Atlántica. Imprenta La Jornada. CEPAS, San José, Costa Rica. 
Sáenz, A. (1978) Historia de la Facultad de Agronomia (1926-1976). Universidad de Costa Rica, San José. Costa Rica.

Sáenz, A. (1985) Historia de la Educación Superior Agricola de Costa Rica (1821-1985). Universidad de Costa Rica, San José, Costa Rica.

Trejos, R. (1998) "La Nueva Institucionalidad para el Desarrollo Rural: Consideraciones para su Construcción”. En Perspectivas Rurales 4, Año 2, N².

Vermeer, R. (1990) El cambio en la agricultura. Litografía COMARFIL. CENAP/UNA, San José, Costa Rica.

Villasuso, J.M. (1992) Cambio Estructural y Rejormas Institucionales en el Sector Agropecuario de América Latina y El Caribe. IICA, San José. Costa Rica.

\section{Periódicos}

-La Nación 6-6-00:18 A. Convenio CNP - Extensión MAG. Un fracaso. Campo pagado.

-La Nación 16-6-00:6 A. Agricultores rechazan propuesta. Gobiemo entregó solución a crisis agropecuaria. Sectores agricolas decidircin hoy qué medidas tomardn para presionar al Gobierno.

-La Nación 28-7-00:4 A. Negociación agricola desde hoy: Levantaron bloqueos anoche.

-La Nación 8-8-00:4 A. Agro suspende negociación. Sin acuerelo reunión con Gobiemo. Exigen freno a cobros judiciales.

-La República 17-10-00:5 A. Réquiem por el pequeño productor: Comisión legislativa dice que reconversión no es suficiente. El $45 \%$ de los fondos de reconversión se ha destinado a ocho proyectos.

-La República 26-10-00:6 A. Frijoleros presentaron amparo. Reclaman competencia desleal. 